\section{Stromal uncertainties in pancreatic cancer}

\section{By Kai-Jye Lou, Senior Writer}

Despite the clear rationale for using hedgehog inhibitors in pancreatic cancer-they deplete the stroma to improve chemotherapy deliveryclinical results have been disappointing. New data suggest that the large amounts of stroma surrounding some pancreatic cancers might actually have protective properties. ${ }^{1,2}$

The findings could explain the negative clinical data for some hedgehog inhibitors in pancreatic cancer, but it remains to be seen whether the cautionary tale applies to other classes of stroma-depleting therapies. In addition, the data provide a rationale to retry two other classes of cancer drugs in pancreatic cancer-angiogenesis inhibitors and immune checkpoint inhibitors (see Box 1, "Revisiting the pancreas").

Pancreatic ductal adenocarcinomas (PDACs) account for the majority of pancreatic cancers. PDACs usually have poor perfusion and vascularization and often are surrounded by abundant stroma, which is thought to supply factors that support tumor growth and hinder drug delivery. ${ }^{3}$

In 2009, an international team led by researchers at Cancer Research UK published preclinical data in Science suggesting that hedgehog pathway inhibitors could deplete the tumor stroma. The group showed that the small molecule smoothened (SMO) inhibitor saridegib (IPI-926) from Infinity Pharmaceuticals Inc. enhanced delivery of the chemotherapeutic gemcitabine and improved survival in mouse models of PDAC. ${ }^{4}$

SMO is a key mediator of hedgehog pathway signaling.

The following year, Infinity began a Phase I/IIb trial of saridegib plus gemcitabine in patients with pancreatic cancer. In January 2012, Infinity stopped the trial after interim data showed that patients receiving the combination had higher rates of progressive disease and lower overall survival than patients receiving placebo plus gemcitabine. The company discontinued development of saridegib that year after interim analyses suggested it also was going to miss the primary endpoint in a Phase II trial in chondrosarcoma and would not meet prespecified criteria for expansion of a Phase II trial in myelofibrosis.

At least two other SMO antagonists are still in clinical trials for pancreatic cancer. LDE225, from Novartis AG, is in a Phase Ib trial in combination with gemcitabine. Erivedge vismodegib, from the
Genentech Inc. unit of Roche, is being tested in combination with various drugs in multiple investigator-led Phase II and Phase I trials.

Interim data from one of those trials showed that Erivedge plus gemcitabine yielded small gains in median progression-free survival (PFS) and overall survival (OS) versus gemcitabine plus placebo. However, patients on the combination had no complete or partial responses and 49 cases of stable disease. There were 3 complete responses, 11 partial responses and 31 cases of stable disease in patients receiving gemcitabine alone.

Genentech markets Erivedge to treat metastatic basal cell carcinoma. The company said that it does not have any clinical trials of Erivedge in pancreatic cancer and declined to comment further.

Novartis did not respond to requests for comment, and Infinity declined to comment.

\section{Stromal uncertainties}

A pair of papers in Cancer Cell could explain the weak showing for SMO inhibitors in pancreatic cancer.

In a study co-led by researchers from the Columbia University Medical Center (CUMC) and Perelman School of Medicine at the University of Pennsylvania, sonic hedgehog homolog (Shh) knockout or chronic treatment with saridegib in a genetic mouse model of PDAC decreased the tumor stroma compared with no alteration or treatment. However, both approaches led to the development of aggressive, poorly differentiated tumors, and treated animals had lower survival rates than controls. In the mouse PDAC model, gemcitabine plus saridegib failed to increase survival compared with gemcitabine alone.

The reason why the findings run counter to the 2009 Science paper likely relates to when the mice started treatment. In the earlier study, researchers began treating the mice after large PDAC tumors developed. Treatment duration was limited to a few weeks because of the short survival time of these animals. In the new study, mice received treatment earlier-they had precancerous pancreatic lesions as opposed to established PDAC tumors.

Kenneth Olive said that the goal of the work published in 2009 was to recapitulate the clinical situation in PDAC, in which most patients are diagnosed with advanced disease. However, he noted that the mice were ill-suited for evaluating longer-term treatment effects as they rapidly succumbed to disease.

Olive is a member of the Herbert Irving Comprehensive Cancer Center at CUMC and an assistant professor of medicine and pathology at CUMC. He was the lead author on the 2009 Science paper and co-corresponding author on the current CUMC-UPenn study.

Olive said that his group started trying to figure out why saridegib failed in pancreatic cancer within hours after Infinity announced the trial halt. The team homed in on the possibility of long-term treatment effects several months later. 


\section{Box 1. Revisiting the pancreas.}

The insights into the nature of these aggressive, poorly differentiated pancreatic tumors suggest a niche for two other classes of cancer treatments-angiogenesis inhibitors and immune checkpoint inhibitors.

Additional data published in the Columbia University Medical Center-Perelman School of Medicine at the University of Pennsylvania study showed that the poorly differentiated tumors in sonic hedgehog homolog (Shh)-deficient or saridegibtreated mice were more vascularized than those from nondeficient mice or vehicle-treated controls. ${ }^{1}$ In the Shh ${ }^{-1-}$ mice, a VEGF receptor-blocking $\mathrm{mAb}$ increased survival compared with IgG control.

Ben Stanger, an assistant professor of medicine at the Perelman School of Medicine, noted that even though antiangiogenic therapies have failed in the overall PDAC patient population, the mouse data in his study suggest that the subset of patients with poorly differentiated tumors could benefit.

"In PDAC, about $10 \%$ of the patients have poorly differentiated tumors, and based on what we've seen in our mouse studies, such tumors might also have higher blood vessel density and be more dependent on the vascular network. If this is the case, it might mean that such tumors will be responsive to antiangiogenic therapies," he said.

Meanwhile, The University of Texas MD Anderson Cancer Center researchers showed that depletion of actin $\alpha 2$ smooth aorta muscle (Acta2; $\alpha$-Sma)-positive myofibroblasts in the mouse PDAC model resulted in a more immunosuppressive tumor microenvironment and increased expression of the immune checkpoint protein Ctla-4 (Cd152) compared with no alteration. ${ }^{2}$ In the myofibroblast-depleted mice, an anti-CTLA4 mAb decreased PDAC progression and increased survival compared with IgG control.

Raghu Kalluri, chair of the Department of Cancer Biology at MD Anderson, noted that the additional data from his group suggest that stroma depletion could create an immune profile in PDAC tumors that renders them sensitive to inhibitors against CTLA-4 and other immune checkpoint proteins. He said that stratifying patients based on their stroma could help identify those that would benefit most from such therapies.

Data from a Phase II trial published in 2010 showed that monotherapy with the anti-CTLA-4 mAb Yervoy ipilimumab was ineffective in advanced PDAC. ${ }^{7}$ Bristol-Myers Squibb Co. markets Yervoy to treat unresectable or metastatic melanoma. $-K-J L$

The results of the CUMC-UPenn study were corroborated in a separate study in PDAC mice led by researchers at The University of Texas MD Anderson Cancer Center. The Texas group showed that depletion of the major subset of hedgehog-responsive cells in the tumor stroma resulted in decreased survival and the development of aggressive, poorly differentiated tumors. Specifically, the subset was myofibroblasts that were positive for actin $\alpha 2$ smooth aorta muscle (ACTA2; $\alpha$-SMA).

The MD Anderson-led group also analyzed tumor samples from a cohort of 53 patients with PDAC and showed that low levels of $\alpha$-SMA were associated with decreased overall survival $(p=0.0053)$.

"Our original hypothesis had been that if we eliminated stromal fibroblasts, we would decrease cancer progression and metastasis, but the results we got were the complete opposite of that," said Raghu Kalluri, a professor and chair of the Department of Cancer Biology at MD Anderson and corresponding author on the MD Anderson study.

"I think these new data mean we need to be cognizant of the

"The current studies suggest the consequences on tumor biology from long-term stroma depletion outweigh the delivery benefit."

-Ben Stanger,

Perelman School of Medicine at the University of Pennsylvania possibility that some classes of anti-stromal compounds have the potential to make pancreatic cancers more aggressive and that the effects you see in the mouse over days and weeks may not be the same as what you see over the longer term," Olive told SciBX.

"The past work has suggested that if you briefly deplete stroma, you could get benefit from improved drug delivery, but the current studies suggest the consequences on tumor biology from long-term stroma depletion outweigh the delivery benefit," added Ben Stanger, an assistant professor of medicine at the Perelman School of Medicine and the other co-corresponding author on the CUMC-UPenn study.

David Tuveson cautioned against generalizing the observations to other classes of stroma-depleting therapies being developed for pancreatic cancer.

"It is not yet known whether going after other targets in the tumor stroma would have the same effect as depletion of stromal fibroblasts or hedgehog inhibition," said Tuveson. He is director of the Lustgarten Foundation Pancreatic Cancer Research Laboratory, a professor at Cold Spring Harbor Laboratory and was senior author on the 2009 Science paper.

Edward Kim, an assistant professor of medicine at the University of California, Davis Comprehensive Cancer Center, said that the current data raise the question of whether the detrimental effects of targeting stromal cells is due to depletion of the stroma itself or other effects. "Attacking the stromal cells results in decreased stroma, but this clearly leads to many more changes than just decreased stroma. These results show targeting stromal cells may not be the answer but fail to elucidate whether removing the acellular stroma may still provide benefit by improving drug delivery," he said.

At least one company is developing a stroma-depleting therapy in pancreatic cancer that goes after an acellular stromal component. Last year, Halozyme Therapeutics Inc. reported data from the Phase Ib portion of a Phase I/II trial in which i.v. PEGPH20 plus gemcitabine resulted in an overall response rate of $42 \%$ in 24 evaluable patients. In a post hoc subgroup analysis, patients with high levels of tumorassociated hyaluronan showed the greatest survival benefit, with double the PFS and triple the OS of patients with low levels of tumor-associated hyaluronan. 


\section{ANALYSIS}

Hyaluronan is a glycosaminoglycan found throughout the body and a component of the tumor stroma in PDAC.

PEGPH20 is a recombinant human PH20 hyaluronidase (sperm adhesion molecule 1; SPAM1; PH20) enzyme conjugated to polyethylene glycol (PEG). Hyaluronidase digests hyaluronan to decrease interstitial fluid pressure around the tumor, leading to improved blood flow and drug delivery.

Halozyme VP and CSO H. Michael Shepard thinks that the negative effects described in the Cancer Cell papers are a cautionary tale for new therapies that target myofibroblasts but probably not for PEGPH20.

He said that the roles of hyaluronan and myofibroblasts in the tumor stroma are very distinct, citing recent studies showing that PEGPH20mediated depletion of hyaluronan plus gemcitabine increased survival in the same mouse PDAC models. ${ }^{5,6}$

\section{New insights}

Kalluri said that his group is now working to elucidate the role of various stromal cell populations in PDAC.

"There are multiple types of fibroblasts in the pancreatic cancer stroma, so we want to determine whether all stromal fibroblasts have a protective role or just the $\alpha$-SMA fibroblasts. We also want to determine what various other cellular populations in the stroma

"Without a better understanding of what the stroma is doing in patients, we can't really determine where anti-stromal therapies will be helpful and where they could be harmful."

-Mert Erkan,

Technical University Munich

Pancreatic Surgery at Technical University Munich.

Erkan noted that the tumor stroma in patients with pancreatic cancer is very heterogeneous-both in amount and composition-and that the consequences of such differences are unclear.

The findings reported in the two new studies are not patented.

Lou, K.-J. SciBX 7(23); doi:10.1038/scibx.2014.665

Published online June 12, 2014

\section{REFERENCES}

1. Rhim, A.D. et al. Cancer Cell; published online May 22, 2014; doi:10.1016/j.ccr.2014.04.021

Contact: Ben Z. Stanger, Perelman School of Medicine at the University of Pennsylvania, Philadelphia, $\mathrm{Pa}$.

e-mail: bstanger@exchange.upenn.edu

Contact: Kenneth P. Olive, Columbia University Medical Center, New York, N.Y.

e-mail: kenolive@columbia.edu

2. Özdemir, B.C. et al. Cancer Cell; published online May 22, 2014; doi:10.1016/j.ccr.2014.04.005

Contact: Raghu Kalluri, The University of Texas MD Anderson Cancer Center, Houston, Texas e-mail: rkalluri@mdanderson.org

3. Erkan, M. et al. Nat. Rev. Gastroenterol. Hepatol. 9, 454-467 (2012)

4. Olive, K.P. et al. Science 324, 1457-1461 (2009)

5. Jacobetz, M.A. et al. Gut 62, 112-120 (2013)

6. Provenzano, P.P. et al. Cancer Cell 21, 418-429 (2012)

7. Royal, R.E. et al. J. Immunother. 33, 828-833 (2010)

\section{COMPANIES AND INSTITUTIONS MENTIONED}

Bristol-Myers Squibb Co. (NYSE:BMY), New York, N.Y. Cancer Research UK, London, U.K. Cold Spring Harbor Laboratory, Cold Spring Harbor, N.Y. Columbia University Medical Center, New York, N.Y. Genentech Inc., South San Francisco, Calif. Halozyme Therapeutics Inc. (NASDAQ:HALO), San Diego, Calif. Infinity Pharmaceuticals Inc. (NASDAQ:INFI), Cambridge, Mass. Lustgarten Foundation, New York, N.Y.

Novartis AG (NYSE:NVS; SIX:NOVN), Basel, Switzerland Roche (SIX:ROG; OTCQX:RHHBY), Basel, Switzerland Perelman School of Medicine at the University of Pennsylvania, Philadelphia, $\mathrm{Pa}$.

Technical University Munich, Munich, Germany University of California, Davis Comprehensive Cancer Center, Davis, Calif.

The University of Texas MD Anderson Cancer Center, Houston, Texas 